\title{
FIRST VERY LOW FREQUENCY DETECTION OF SHORT REPEATED BURSTS FROM MAGNETAR SGR J1550-5418
}

\author{
Y. T. Tanaka ${ }^{1}$, Jean-Pierre Raulin ${ }^{2}$, Fernando C. P. Bertoni ${ }^{2}$, P. R. Fagundes ${ }^{3}$, J. Chau $^{4}$, N. J. Schuch ${ }^{5}$, M. Hayakawa ${ }^{6}$,

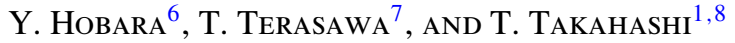 \\ ${ }^{1}$ Institute of Space and Astronautical Science, Japan Aerospace Exploration Agency, Japan; tanaka@ astro.isas.jaxa.jp \\ ${ }^{2}$ CRAAM, Presbyterian Mackenzie University, São Paulo, Brazil \\ ${ }^{3}$ University of Vale do Paraiba, São José dos Campos, Brazil \\ ${ }^{4}$ Radio Observatorio de Jicamarca, Instituto Geofísico del Perú, Lima, Perú \\ ${ }^{5}$ INPE's Southern Regional Space Research Center, Santa Maria, Brazil \\ ${ }^{6}$ University of Electro-Communications, Japan \\ ${ }^{7}$ Institute for Cosmic Ray Research, University of Tokyo, Japan \\ ${ }^{8}$ University of Tokyo, Japan \\ Received 2010 June 21; accepted 2010 July 27; published 2010 August 27
}

\begin{abstract}
We report on the first detection of ionospheric disturbances caused by short repeated gamma-ray bursts from the magnetar SGR J1550-5418. Very low frequency (VLF) radio wave data obtained in South America clearly show sudden amplitude and phase changes at the corresponding times of eight soft gamma-ray repeater bursts. Maximum amplitude and phase changes of the VLF signals appear to be correlated with the gamma-ray fluence. On the other hand, VLF recovery timescales do not show any significant correlation with the fluence, possibly suggesting that the bursts' spectra are not similar to each other. In summary, Earth's ionosphere can be used as a very large gamma-ray detector and the VLF observations provide us with a new method to monitor high-energy astrophysical phenomena without interruption such as Earth occultation.
\end{abstract}

Key words: stars: individual (SGR J1550-5418) - stars: neutron

Online-only material: color figures

\section{INTRODUCTION}

Very low frequency (VLF; 3-30 kHz) radio waves are reflected at Earth's lower ionosphere and ground, and propagate within the Earth-ionosphere waveguide (e.g., Wait 1962). Since amplitude and phase of VLF radio waves are sensitive to the condition of the lower ionosphere, they have been utilized to investigate the physics of the lower ionosphere. Energetic electrons can precipitate into the ionosphere due to wave-particle interaction in the magnetosphere and cause VLF signal amplitude and phase perturbations (e.g., Kikuchi \& Evans 1983). Soft X-rays from solar flares are also another source of ionospheric disturbances, which are detected using VLF signals (e.g., Todoroki et al. 2007; Raulin et al. 2010).

Besides these solar-terrestrial events, the lower ionosphere is also affected by high-energy photons (X-rays and gamma rays) from extra-solar sources. An ionospheric disturbance caused by a cosmic gamma-ray burst was first reported by Fishman \& Inan (1988). It suggested that gamma rays deposit their energies in the lower ionosphere, abnormally ionize the neutral atmosphere there, and modify the electron density height profile. In addition, it is known that giant flares from soft gamma-ray repeaters (SGRs, also called magnetars) significantly affect the lower ionosphere (Inan et al. 1999, 2007; Tanaka et al. 2008).

Magnetars emit a lot of short-duration gamma-ray flares repeatedly during active phases (e.g., Woods \& Thompson 2006). Typical duration and flux of the short repeated bursts are $0.1-1 \mathrm{~s}$ and $10^{-6} \mathrm{erg} \mathrm{s}^{-1} \mathrm{~cm}^{-2}$, respectively. Furthermore, magnetars rarely emit exceptionally bright gamma-ray flares (giant flares). So far, only three giant flares were recorded. The first one was detected in 1979, from the source SGR 0526-66 (Mazets et al. 1979). The second and third ones were emitted by SGR 1900+14 and SGR 1806-20, and they were observed by satellites in 1998 (Hurley et al. 1999; Mazets et al. 1999; Tanaka et al. 2007) and 2004 (Terasawa et al. 2005; Hurley et al. 2005; Palmer et al. 2005; Frederiks et al. 2007), respectively. Since the fluences were much larger than those of GOES X-class solar flares by a few orders of magnitude, ionospheric disturbances caused by these giant flares were detected as sudden and large amplitude changes of VLF radio waves (Inan et al. 1999, 2007; Tanaka et al. 2008).

On the other hand, VLF amplitude and phase changes caused by short repeated gamma-ray bursts from a magnetar have not been detected so far because of the lack of high sensitivity of VLF observing systems. On 2009 January 22, one of the known magnetars SGR J1550-5418 emitted a lot of short-duration gamma-ray bursts repeatedly (Mereghetti et al. 2009). In this Letter, we report on the first VLF detection of short repeated gamma-ray flares from this object. VLF data were provided by the South America VLF Network (SAVNET) tracking system (Raulin et al. 2009). In Section 2, we describe details of the SAVNET observations. Comparison of VLF amplitude and phase changes with gamma-ray fluences measured by INTEGRAL satellite are presented and discussed in Sections 3 and 4, respectively. We summarize this Letter in Section 5.

\section{OBSERVATIONS}

The VLF data shown in this Letter were obtained by SAVNET, which was recently installed in Brazil, Peru, and Argentina (see Raulin et al. (2009) for the details of the SAVNET instrumental facility). Figure 1 shows locations of a relevant observing station ATI (Atibaia, São Paulo, Brazil) as well as five VLF transmitters (NPM, NLK, NDK, NAU, and NAA), and VLF signals from them have been continuously recorded with the time resolution 


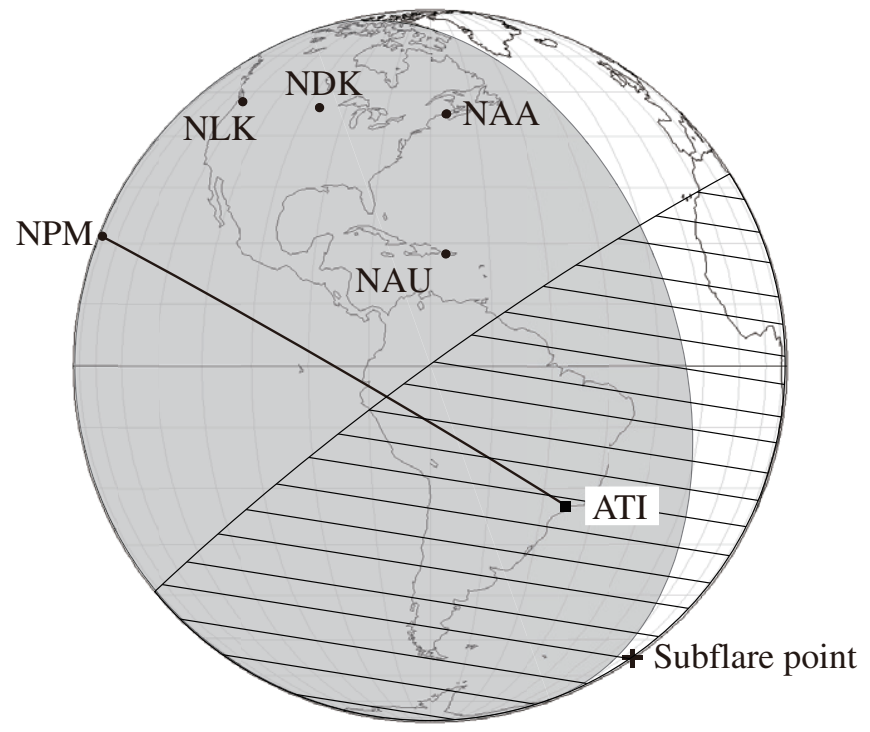

Figure 1. VLF propagation path from NPM transmitter (Hawaii) to ATI observing station (São Paulo, Brazil). Also shown are the locations of other four VLF transmitters (NLK, NDK, NAA, and NAU). Shaded hemisphere indicates the nightside part of the Earth at 6:48 UT, when the largest burst occurred (see Table 1). The part of the Earth illuminated by gamma rays at 6:48 UT is also drawn by dashed area.

of $1 \mathrm{~s}$. The propagation path from NPM $(21.4 \mathrm{kHz})$ to ATI is also drawn in Figure 1. Shaded hemisphere in Figure 1 exhibits the nightside part of the Earth at 6:48 UT, when the most intense gamma-ray flare occurred. The point on the Earth directly beneath the flare (subflare point) was located at $54.3 \mathrm{~S}$, $14.0 \mathrm{E}$, and its position is shown using a cross. The part of the Earth illuminated by gamma rays at 6:48 UT is illustrated by dashed area.

Figure 2 shows NPM-ATI amplitude and phase data recorded from 4:00 UT to 10:00 UT on 2009 January 22. In Figure 3, we also display an extended view of NPM-ATI data together with the gamma-ray light curve ${ }^{9}$ observed by INTEGRAL satellite around 6:48 UT (Mereghetti et al. 2009). Due to the high sensitivity of the SAVNET facility, we can clearly see rapid amplitude and phase changes at the corresponding times of the short repeated bursts from SGR J1550-5418. Therefore, we can robustly claim that the rapid changes were caused by the short gamma-ray bursts from the magnetar. We listed in Table 1 the properties of the SGR short bursts detected by the NPMATI VLF propagation path. Although these magnetar bursts were detected at other SAVNET receiving stations, like PAL (Palmas, TO, Brazil), SMS (São Martinho da Serra, RS, Brazil), PIU (Piura, Peru), and EACF (Antarctica), in this short Letter we concentrate on the records from ATI receiving station. A detailed comparison of VLF phases and amplitudes observed by the other receivers is out of the scope of this Letter and will be reported in a subsequent forthcoming article.

\section{ANALYSIS}

To investigate the influence of gamma-ray irradiation in the lower ionosphere, we need to characterize the VLF amplitude and phase data. To do this, we estimated the maximum amplitude $(\Delta A)$ and phase variations $(\Delta \phi)$ as well as the recovery timescales of the amplitude as follows. First, we extracted the

\footnotetext{
9 http://www.isdc.unige.ch/integral/ibas/cgi-bin/ibas_acs_web.cgi
}

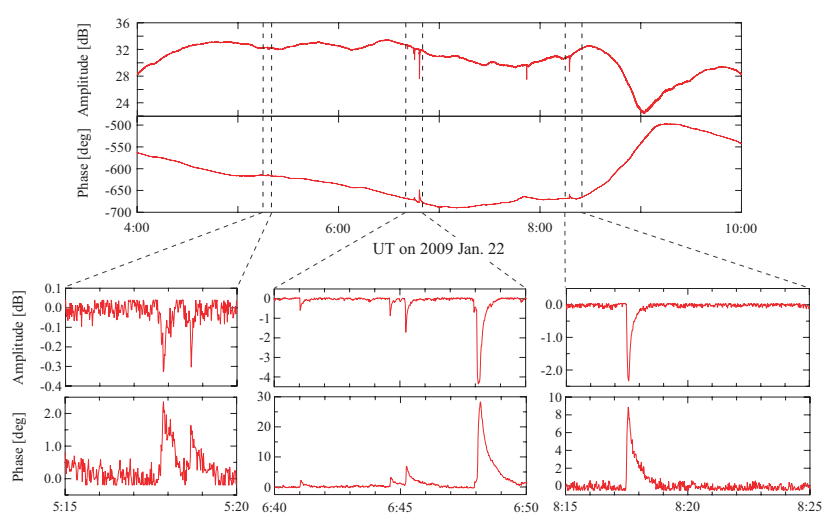

Figure 2. Amplitude and phase variations of a VLF signal from NPM transmitter $(21.4 \mathrm{kHz}$ ), which were observed at ATI (see Figure 1) from 4:00 UT to 10:00 UT on 2009 January 22. Lower figures are background-subtracted blownups at time ranges during which short repeated SGR bursts were detected (see also Table 1).

(A color version of this figure is available in the online journal.)

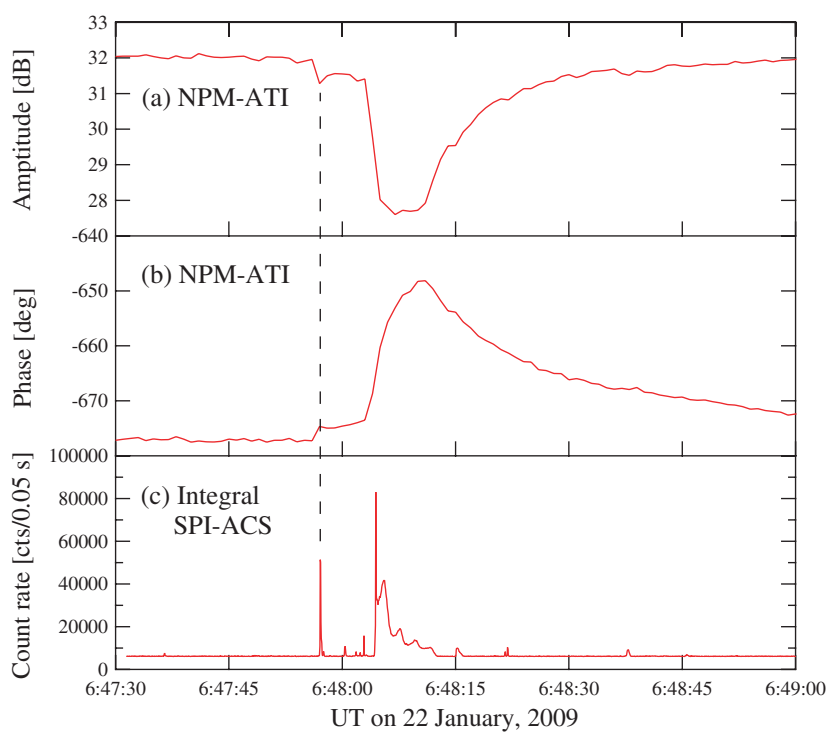

Figure 3. (a) Blown-up VLF amplitude data from the NPM-ATI path around 6:48 UT. The vertical dashed line shows the time 6:47:57.1 UT, when a relatively large gamma-ray flare was observed by INTEGRAL (see also Table 1). (b) Same as (a), but for VLF phase data. (c) INTEGRAL/SPI-ACS light curve around 6:48 UT. Note that the peak of the brightest burst at 6:48:04.3 UT was probably higher than shown here, due to a saturation problem for high count rates (Mereghetti et al. 2009)

(A color version of this figure is available in the online journal.)

data before and after each burst, and chose a proper functional form to represent the baseline level. Most of the baseline levels can be well fitted by first-order polynomial functions. When the baseline levels showed curvature, we used second-order polynomial functions to represent them. After subtracting the trends from the amplitude and phase data, we obtained $\Delta A$ and $\Delta \phi$, which are tabulated in Table 1 . To estimate the typical errors of $\Delta A$ and $\Delta \phi$, we made histograms of the residuals, which were distributed around 0 with a Gaussian-like form. Therefore, we fitted the histogram using a Gaussian and took the variance as a typical error.

To quantify the recovery timescales of the VLF amplitude data, we have used the function

$$
\begin{aligned}
f(t)= & (\text { Baseline level }) \\
& -\frac{F_{0}}{\left\{\exp \left(\left(t_{0}-t\right) / t_{\mathrm{fall}}\right)+\exp \left(\left(t-t_{0}\right) / t_{\mathrm{rcv}}\right)\right\}},
\end{aligned}
$$


Table 1

Short Repeated Bursts from SGR J1550-5418 Detected by NPM-ATI VLF Data

\begin{tabular}{|c|c|c|c|c|c|c|c|}
\hline $\begin{array}{l}\text { Start UT } \\
\text { on Jan } 22^{\mathrm{a}}\end{array}$ & $\begin{array}{c}\text { INTEGRAL } \\
\text { ID }^{\mathrm{a}}\end{array}$ & $\begin{array}{l}\text { Duration }^{\mathrm{a}} \\
\text { (s) }\end{array}$ & $\begin{array}{c}\text { Gamma-ray Fluence }{ }^{\mathrm{a}, \mathrm{b}} \\
10^{-5}\left(\mathrm{erg} \mathrm{cm}^{-2}\right)\end{array}$ & $\begin{array}{c}\text { Amplitude } \\
\text { Change }(\mathrm{dB})\end{array}$ & $\begin{array}{c}\text { Phase } \\
\text { Change (deg) }\end{array}$ & $\begin{array}{c}\text { Recovery Timescale } \\
\text { of Amplitude (s) }\end{array}$ & $\begin{array}{c}\text { Lowering of } \\
\text { Reflection Height }(\mathrm{km})\end{array}$ \\
\hline 51751.7 & 85 & 0.45 & $1.91 \pm 0.01$ & $-0.33 \pm 0.03$ & $2.5 \pm 0.3$ & $7.1 \pm 1.2$ & $8.0 \pm 1.0$ \\
\hline 51839.5 & 93 & 1.00 & $1.44 \pm 0.01$ & $-0.32 \pm 0.03$ & $2.0 \pm 0.3$ & $2.0 \pm 0.7$ & $6.4 \pm 1.0$ \\
\hline 64102.1 & 108 & 1.00 & $2.35 \pm 0.01$ & $-0.59 \pm 0.04$ & $2.2 \pm 0.3$ & $6.3 \pm 0.6$ & $4.5 \pm 0.6$ \\
\hline 64436.4 & 117 & 1.75 & $1.93 \pm 0.01$ & $-0.91 \pm 0.05$ & $2.8 \pm 0.3$ & $5.4 \pm 0.6$ & $5.8 \pm 0.6$ \\
\hline 64513.9 & 121 & 1.45 & $>4.59 \pm 0.01$ & $-1.8 \pm 0.05$ & $6.5 \pm 0.3$ & $5.9 \pm 0.3$ & $13 \pm 0.6$ \\
\hline 64757.1 & 141 & 0.35 & $1.82 \pm 0.01$ & $-0.74 \pm 0.04$ & $2.2 \pm 0.5$ & $\ldots{ }^{c}$ & $4.5 \pm 1.0$ \\
\hline 64804.3 & 149 & 8.15 & $>27.76 \pm 0.03$ & $-4.4 \pm 0.04$ & $29 \pm 0.5$ & $9.7 \pm 0.2$ & $60 \pm 1$ \\
\hline 81729.4 & 176 & 6.20 & $6.59 \pm 0.02$ & $-2.4 \pm 0.1$ & $9.3 \pm 0.3$ & $12.0 \pm 0.4$ & $14 \pm 0.5$ \\
\hline
\end{tabular}

Notes.

a Taken from Mereghetti et al. (2009).

b The energy range is $25 \mathrm{keV}$ to $2 \mathrm{MeV}$.

${ }^{c}$ Unable to determine by fitting.

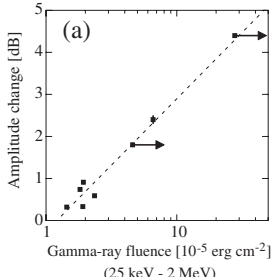

$(25 \mathrm{keV}-2 \mathrm{MeV})$

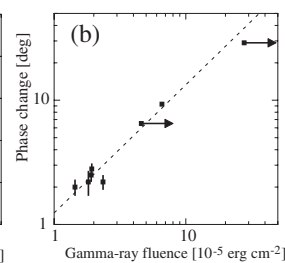

$(25 \mathrm{keV}-2 \mathrm{MeV})$

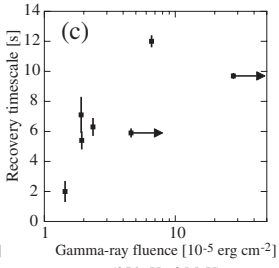

$(25 \mathrm{keV}-2 \mathrm{MeV})$
Figure 4. (a) Relation between observed amplitude changes and gamma-ray fluences ( $25 \mathrm{keV}$ to $2 \mathrm{MeV}$ ) measured by INTEGRAL satellite (Mereghetti et al. 2009). Values are tabulated in Table 1. (b) Same as (a), but for observed phase changes. (c) Same as (a), but for recovery timescales estimated by fitting.

where $F_{0}$ is a typical amplitude decrease, $t_{\text {fall }}$ is a falling time, and $t_{\text {rcv }}$ is a recovery timescale. We fitted the data using this function and determined $t_{\mathrm{rcv}}$ for each burst (see Table 1).

\section{DISCUSSION}

\subsection{Amplitude and Phase Changes}

In Figures 4(a) and (b), we plot $\Delta A$ and $\Delta \phi$ against the gammaray fluence from $25 \mathrm{keV}$ to $2 \mathrm{MeV}$ for each burst (see also Table 1). Although there are not many data points, possible correlations between $\Delta A$ and $\Delta \phi$, and gamma-ray fluences are seen. We note that similar correlations were also reported in the case of X-ray solar flares (McRae \& Thomson 2004; Pacini \& Raulin 2006).

We can understand these evidences of correlation in terms of the lowering of the reflection height due to gamma-ray ionization. Under a typical undisturbed nighttime condition, VLF waves are thought to be reflected at $\sim 85 \mathrm{~km}$ (e.g., Carpenter et al. 1997). When gamma rays are directed onto the Earth, they deposit most of their energy in the lower ionosphere, ionize the neutral atmosphere there, and produce free electrons. The typical altitude where these free electrons are produced depends on the photon energy. For example, by using Monte Carlo simulation Inan et al. (1999) reported that $3 \mathrm{keV}$ and $10 \mathrm{keV}$ photons mainly ionize the atmosphere at $\sim 82 \mathrm{~km}$ and $60 \mathrm{~km}$, respectively. Similar calculations have shown that gamma-ray illumination increases electron number density below $\sim 85 \mathrm{~km}$, depending on the photon spectrum (e.g., Brown 1973; Baird 1974; Tanaka et al. 2008). Consequently, the VLF radio waves are reflected at a lower altitude than usual, and hence the phase of propagating VLF waves is advanced.

Due to the lack of observation, the exact photon spectrum for each burst was not reported so far. But following Mereghetti et al. (2009), we assume the spectral shape of an optically thin thermal bremsstrahlung with $k T=40 \mathrm{keV}$. Then, the number of higher-energy photons increases as the fluence goes up. Higherenergy photons can penetrate deeper at low altitude and increase the electron number density there. As a result, the reflection height becomes lower as the gamma-ray fluence increases.

By treating the propagation of VLF radio waves using the mode theory (Wait \& Spies 1964), we estimated the reduction of the reflection height $\Delta H$ from the phase change $\Delta \phi$. In the following, we have assumed that the lower ionosphere is isotropic and a sharply bounded medium (Wait \& Spies 1964), and we have used a phase velocity expression given by Wait (1959). Then, the relation between $\Delta \phi$ and $\Delta H$ can be expressed as (Inan \& Carpenter 1987)

$$
\frac{\Delta \phi}{d \Delta H} \simeq-\frac{2 \pi f}{h c}\left[\frac{h}{2 R_{\mathrm{e}}}+C_{n}^{2}\right] \quad C_{n}=\frac{(2 n-1) \lambda}{4 h},
$$

where $d$ is the length of the disturbed region along the great circle path, $f$ is the wave frequency, $h$ is the typical reflection height, $c$ is the speed of light, $R_{\mathrm{e}}$ is Earth's radius, $\lambda$ is the wavelength of the VLF radio wave, and $n$ is the order of the waveguide mode. For a long propagation distance and a normal nighttime reflection height of $h \sim 85 \mathrm{~km}$, Wait \& Spies (1964) showed that the second mode $(n=2)$ would be dominant. Therefore, we were able to calculate $\Delta H$ from the observed $\Delta \phi$ for each burst and these values are tabulated in Table 1 . We note that $\Delta H$ calculated by using Equation (2) is a rough estimate, and Monte Carlo simulations are required to obtain more accurate values. Nonetheless, this gross estimation would be meaningful to qualitatively consider the effect of gamma-ray illumination.

Next, we consider a mechanism for the observed decrease of VLF wave amplitude during the gamma-ray illumination. We can understand it on the basis of the altitude dependence of the collision frequency $v_{\mathrm{e}}$ between electrons and neutral atoms. $v_{\mathrm{e}}$ is higher for lower altitudes and is often modeled as $v_{\mathrm{e}}=1.816 \times 10^{11} \exp (-0.15 z)$, where $z$ is the altitude measured in $\mathrm{km}$ (e.g., Wait \& Spies 1964). Consequently, as the reflection height becomes lower, VLF radio waves are more attenuated, and hence their amplitudes decrease.

These hints of correlation suggest that it would be possible to deduce a gamma-ray fluence from $\Delta A$ and $\Delta \phi$. We note that these relations are applicable only for this particular VLF frequency $(21.4 \mathrm{kHz})$. There are also another uncertainties which might affect $\Delta A$ and $\Delta \phi$ such as the altitude profile of the ambient electron number density. Nonetheless, we claim that Earth's 
ionosphere can be used as a new gamma-ray "detector" and VLF data can provide a unique information on incident gamma-ray fluences, even if satellites in space were not able to observe it. Therefore, we stress here that this VLF method is a new potential technique for monitoring high-energy transient phenomena in the universe, once we know in advance which source is active.

\subsection{Recovery Timescale}

We plot in Figure 4(c) the recovery timescale of each burst against the gamma-ray fluence. We did not find any significant correlation between the two quantities, and all the recovery timescales were in the range of $2-12 \mathrm{~s}$. Since the $t_{\text {rcv }}$ are longer than the burst durations (see Table 1), the observed VLF amplitude and phase time profiles are different from the gammaray light curves. We also fitted the phase data with a similar function of Equation (1) and calculated the $t_{\mathrm{rcv}}$. Again, we did not find any significant correlation between gamma-ray fluences and recovery timescales.

As shown in Figure 2, recovery time profiles can be well represented by an exponential-like function of a single parameter $t_{\text {rcv }}$. On the other hand, in the case of magnetar giant flares, two different recovery timescales were reported (Inan et al. 2007; Tanaka et al. 2008). Namely, an initial rapid recovery of a few seconds was followed by a long enduring recovery lasting for $>1 \mathrm{hr}$. The long-duration recovery is interpreted as due to the neutralization of positive and negative ions at an altitude below $60 \mathrm{~km}$ (Inan et al. 2007), which means that ionization by gamma rays mainly occurred at such a low altitude. In fact, it is known that the photon spectrum and gamma-ray fluence of giant flares are much harder and higher than those of short repeated bursts (Woods \& Thompson 2006). Lack of such a long-duration recovery in our VLF data suggests that the spectra of short repeated bursts were relatively soft compared to that of giant flares.

As shown above, we interpreted the VLF amplitude and phase changes as due to the lowering of the reflection height. In this case, faster recovery timescales are expected for larger gammaray fluences, because the electron attachment rate is a negative function of altitude (Rowe et al. 1974). However, as shown in Figure 4(c), we did not find such a trend. This might be due to different spectrum for each burst, contrary to what we have assumed in this Letter following Mereghetti et al. (2009). The harder the spectrum, the lower the reflection height and the faster the recovery time. Monte Carlo simulations are required to confirm this possibility, and the results will be reported in a subsequent article.

\section{SUMMARY}

We have detected, for the first time, ionospheric disturbances caused by short repeated gamma-ray bursts from a magnetar.
Amplitude and phase changes of VLF propagating waves are correlated with gamma-ray fluences. This can be understood in terms of the lowering of the reflection height. While satellites in space cannot continuously observe the whole sky due to Earth occultation, Earth's ionosphere can monitor it without interruption. VLF observations provide us with a new method to monitor high-energy transient phenomena of astrophysical importance.

Y.T.T. is supported by a JSPS Research Fellowship for Young Scientists. J.P.R. thanks FAPESP (Proc. 06/02979-0), CNPq (Proc. 304433/2004-7), and MACKPESQUISA funding agencies.

\section{REFERENCES}

Baird, G. A. 1974, J. Atmos. Terr. Phys., 36, 1565

Brown, R. T. 1973, Nature, 246, 83

Carpenter, D. L., et al. 1997, J. Geophys. Res., 102, 347

Fishman, G. J., \& Inan, U. S. 1988, Nature, 331, 418

Frederiks, D. D., Golenetskii, S. V., Palshin, V. D., Aptekar, R. L., Ilyinskii, V. N., Oleinik, F. P., Mazets, E. P., \& Cline, T. L. 2007, Astron. Lett., 33,

Hurley, K., et al. 1999, Nature, 397, 41

Hurley, K., et al. 2005, Nature, 434, 1098

Inan, U. S., \& Carpenter, D. L. 1987, J. Geophys. Res., 92, 3293

Inan, U. S., Lehtinen, N. G., Lev-Tov, S. J., Johnson, M. P., Bell, T. F., \& Hurley, K. 1999, Geophys. Res. Lett., 26, 3357

Inan, U. S., Lehtinen, N. G., Moore, R. C., Hurley, K., Boggs, S., Smith, D. M., \& Fishman, G. J. 2007, Geophys. Res. Lett., 34, 8103

Kikuchi, T., \& Evans, D. S. 1983, J. Geophys. Res., 88, 871

Mazets, E. P., Cline, T. L., Aptekar', R. L., Butterworth, P. S., Frederiks, D. D., Golenetskii, S. V., Il'Inskii, V. N., \& Pal'Shin, V. D. 1999, Astron. Lett., 25, 635

Mazets, E. P., Golentskii, S. V., Ilinskii, V. N., Aptekar, R. L., \& Guryan, I. A. 1979, Nature, 282, 587

McRae, W. M., \& Thomson, N. R. 2004, J. Atmos. Sol.-Terr. Phys., 66, 77

Mereghetti, S., et al. 2009, ApJ, 696, L74

Pacini, A. A., \& Raulin, J. P. 2006, J. Geophys. Res. (Space Phys.), 111, 9301

Palmer, D. M., et al. 2005, Nature, 434, 1107

Raulin, J. P., Correia de Matos David, P., Hadano, R., Saraiva, A. C. V., Correia, E., \& Kaufmann, P. 2009, Earth Moon Planets, 104, 247

Raulin, J. P., et al. 2010, J. Geophys. Res., 115, A07301

Rowe, J. N., Mitra, A. P., Ferraro, A. J., \& Lee, H. S. 1974, J. Atmos. Terr. Phys., 36, 755

Tanaka, Y. T., Terasawa, T., Kawai, N., Yoshida, A., Yoshikawa, I., Saito, Y., Takashima, T., \& Mukai, T. 2007, ApJ, 665, L55

Tanaka, Y. T., Terasawa, T., Yoshida, M., Horie, T., \& Hayakawa, M. 2008, J. Geophys. Res. (Space Phys.), 113, A07307

Terasawa, T., et al. 2005, Nature, 434, 1110

Todoroki, Y., Maekawa, S., Yamauchi, T., Horie, T., \& Hayakawa, M. 2007, Geophys. Res. Lett., 34, 3103

Wait, J. R. 1959, Proc. IRE, 47, 998

Wait, J. R. 1962, Electromagnetic Waves in Stratified Media (New York: Elsevier)

Wait, J. R., \& Spies, K. P. 1964, NBS Tech., 300

Woods, P. M., \& Thompson, C. 2006, in Soft Gamma Repeaters and Anomalous X-ray Pulsars: Magnetar Candidates, ed. W. H. G. Lewin \& M. van der Klis (New York: Cambridge Univ. Press), 547 